PROCEEDINGS OF THE

AMERICAN MATHEMATICAL SOCIETY

Volume 130, Number 8, Pages 2397-2402

S 0002-9939(02)06425-0

Article electronically published on February 12, 2002

\title{
NOTIONS OF BOUNDARIES FOR FUNCTION SPACES
}

\author{
RICHARD F. BASENER \\ (Communicated by Mei-Chi Shaw)
}

\begin{abstract}
Sibony and the author independently defined a higher order generalization of the usual Shilov boundary of a function algebra which yielded extensions of results about analytic structure from one dimension to several dimensions. Tonev later obtained an alternative characterization of this generalized Shilov boundary by looking at closed subsets of the spectrum whose image under the spectral mapping contains the topological boundary of the joint spectrum. In this note we define two related notions of what it means to be a higher order/higher dimensional boundary for a space of functions without requiring that the boundary be a closed set. We look at the relationships between these two boundaries, and in the process we obtain an alternative proof of Tonev's result. We look at some examples, and we show how the same concepts apply to convex sets and linear functions.
\end{abstract}

In what follows, let $M$ be a compact Hausdorff space, and let $Y$ be a complex (or real) Banach space. Let $\mathcal{F}$ be a family of continuous functions from $M$ to $Y$. Let $q$ denote a nonnegative integer.

Notation 1. If $S \subseteq \mathcal{F}$, let $V(S)$ denote the $\mathcal{F}$-variety given by

$$
V(S)=\{x \in M \mid \forall f \in S, f(x)=0\} .
$$

Let \#S denote the cardinality of the set $S$.

Definition 1. If $E \subseteq M$, we will say that $E$ is a " $q$ th order boundary" for $\mathcal{F}$ if $\forall S \subseteq \mathcal{F}$ with $\# S \leq q$ and $\forall f \in \mathcal{F}$

$$
\exists x \in E \cap V(S)
$$

such that

$$
\|f(x)\|=\max _{V(S)}\|f\| .
$$

The condition of the definition is, of course, equivalent to saying that $E \cap V(S)$ is a "boundary" in the usual sense for $\left.\mathcal{F}\right|_{V(S)}$ in the case where $\mathcal{F}$ is a uniform algebra or function space.

Definition 2. If $E \subseteq M, E$ is a " $q$-tuple boundary" for $\mathcal{F}$ if

$$
\forall F=\left(f_{1}, \ldots, f_{q}\right): M \rightarrow Y^{q} \text { with } f_{1}, \ldots, f_{q} \in \mathcal{F}, \partial F(M) \subseteq F(E) .
$$

Here " $\partial$ " denotes the topological boundary in $Y^{q}$. If $q=0$, we understand the condition to be vacuous, so that any subset of $M$, including $\emptyset$, is a 0 -tuple boundary for $\mathcal{F}$.

Received by the editors March 23, 2001.

2000 Mathematics Subject Classification. Primary 32Fxx, 46J10.

(C)2002 American Mathematical Society 
Let $\mathcal{A}$ be a uniform algebra with spectrum $M$. Let $\partial_{0} \mathcal{A}$ denote the usual Shilov boundary of $\mathcal{A}$. Recall from the paper of Sibony [5] or from [1] that the $q$ th order generalized Shilov boundary of $\mathcal{A}$ may be defined as

$$
\partial_{q} \mathcal{A}=\text { Closure } \bigcup\left\{\left.\partial_{0} \mathcal{A}\right|_{V(S)}: S \subseteq \mathcal{A}, \# S \leq q\right\},
$$

where $\left.\mathcal{A}\right|_{V(S)}$ is the uniform algebra on $V(S)$ generated by the restriction of the functions from $\mathcal{A}$. It is clear that $\partial_{q} \mathcal{A}$ is a $q$ th order boundary for $\mathcal{A}$ as defined above. Also, if $K$ is a closed $q$ th order boundary for $\mathcal{A}$, then it is easy to see that $\left.K \supseteq \partial_{0} \mathcal{A}\right|_{V(S)}$ for each $S \subseteq \mathcal{A}$ with $\# S \leq q$, so $K \supseteq \partial_{q} \mathcal{A}$. Thus $\partial_{q} \mathcal{A}$ is the smallest closed $q$ th order boundary for $\mathcal{A}$.

The " $q$-tuple boundary" was introduced by Tonev for uniform algebras and for function spaces (Definitions 3.1.2 and 3.2.3 in [8]).

Proposition 1. If $E \subseteq M$ is a q-tuple boundary for $\mathcal{F}$ and if $0 \leq r<q$, then $E$ is an $r$-tuple boundary for $\mathcal{F}$.

If $r=0$ this is trivial, so suppose $r>0$. Let $f_{1}, \ldots, f_{r} \in \mathcal{F}$ and let $F=$ $\left(f_{1}, \ldots, f_{r}\right)$. Let $z_{0} \in \partial F(M) \subseteq Y^{r}$. We must show that $z_{0} \in F(E)$.

Choose $x_{0} \in M$ such that $F\left(x_{0}\right)=z_{0}$. Define $\tilde{F}: M \rightarrow Y^{q}$ by

$$
\tilde{F}=\left(f_{1}, \ldots, f_{r}, f_{1}, \ldots, f_{1}\right),
$$

where $f_{1}$ is repeated $(q-r)$ times at the end. Then clearly

$$
\tilde{F}\left(x_{0}\right) \in \partial \tilde{F}(M),
$$

so by definition of the $q$-tuple boundary $\exists x_{1} \in E$ such that

$$
\tilde{F}\left(x_{1}\right)=\tilde{F}\left(x_{0}\right) \text {. }
$$

But then $F\left(x_{1}\right)=F\left(x_{0}\right)=z_{0}$, as desired.

Proposition 2. If $E$ is a $(q+1)$-tuple boundary for $\mathcal{F}$, then $E$ is a qth order boundary for $\mathcal{F}$.

Let $S \subseteq \mathcal{F}$ with $\# S \leq q$ and let $f \in \mathcal{F}$. Choose $x_{0} \in V(S)$ such that

$$
\left\|f\left(x_{0}\right)\right\|=\max _{V(S)}\|f\| .
$$

Then let $S=\left\{f_{1}, \ldots, f_{r}\right\}$, where $r \leq q$, and define

$$
F=\left(f, f_{1}, \ldots, f_{r}\right): M \rightarrow Y^{r+1},
$$

where $r+1 \leq q+1$. Note that $F\left(x_{0}\right) \in \partial F(M)$, since

$$
(1+1 / n) F\left(x_{0}\right) \in Y^{r+1} \backslash F(M)
$$

for $n=1,2, \ldots$ (If $F\left(x_{0}\right)=0$, consider

$$
(1 / n, 0, \ldots, 0)
$$

instead.) By Proposition 1, $E$ is an $(r+1)$-tuple boundary for $\mathcal{F}$; hence $\exists x_{1} \in E$ such that

$$
F\left(x_{1}\right)=F\left(x_{0}\right)
$$

It follows that $x_{1} \in V(S) \cap E$ and

$$
\left\|f\left(x_{1}\right)\right\|=\left\|f\left(x_{0}\right)\right\|=\max _{V(S)}\|f\|,
$$

as desired. 
That a $q$ th order boundary for $\mathcal{F}$ need not be a $(q+1)$-tuple boundary for $\mathcal{F}$ in the general case (no restrictions on $\mathcal{F}$ ) is easy to see.

Example 1. Let $M=\{z \in \mathbb{C}|1 / 2 \leq| z \mid \leq 1\}$. Let $\mathcal{F}=\{f: M \rightarrow \mathbb{C} \mid f$ is continuous, $f(M) \subseteq M, f$ maps $|z|=1 / 2$ onto $|z|=1 / 2$, and $f$ maps $|z|=1$ onto $|z|=$ $1\}$. Then $\{|z|=1\}$ is a minimal 0 th order boundary for $\mathcal{F}$, but the minimal 1-tuple boundary is $\{|z|=1 / 2\} \cup\{|z|=1\}$.

The next result shows that if we require that $\mathcal{F}$ be a uniform algebra on $M$ and if we consider closed subsets of $M$, the two notions of boundary are equivalent. This result is motivated, as is the second notion of boundary, by the result that if $\mathcal{A}$ is a uniform algebra with spectrum $M$ and if $f \in \mathcal{A}$, then $f\left(\partial_{0} \mathcal{A}\right) \supseteq \partial f(M)$ (compare Theorem 4.3 in Chapter I of [4]). This result was generalized to the higher order Shilov boundary and the topological boundary of the joint spectrum by Tonev [7]. Our proof of the following theorem yields an alternative proof of Tonev's result.

Theorem 1. Let $\mathcal{A}$ be a uniform algebra with spectrum $M$. Let $K \subseteq M$ be a closed qth order boundary for $\mathcal{A}$. Then $K$ is a $(q+1)$-tuple boundary for $\mathcal{A}$.

Proof. Suppose $K$ as above is not a $(q+1)$-tuple boundary for $\mathcal{A}$. Then $\exists F=$ $\left(f_{1}, \ldots, f_{q+1}\right)$ with $f_{1}, \ldots, f_{q+1} \in \mathcal{A}$ and $\exists z_{0} \in \partial F(M) \subseteq \mathbb{C}^{q+1}$ with $z_{0} \notin F(K)$.

Choose $z_{1} \in \mathbb{C}^{q+1} \backslash F(M)$ such that

$$
\left\|z_{0}-z_{1}\right\|<(1 / 3) \operatorname{distance}\left(z_{0}, F(K)\right) .
$$

Without loss of generality, $z_{0}=0$ and $z_{1}=(1,0,0, \ldots, 0)$.

Now $z_{1}$ is not in the joint spectrum of $f_{1}, \ldots, f_{q+1}$; hence $\exists g_{1}, \ldots, g_{q+1} \in \mathcal{A}$ such that

$$
\left(1-f_{1}\right) g_{1}+\left(0-f_{2}\right) g_{2}+\ldots+\left(0-f_{q+1}\right) g_{q+1}=1 .
$$

Since $z_{0} \in \partial F(M) \subseteq F(M), \exists x_{0} \in M$ such that $F\left(x_{0}\right)=z_{0}$. Let $S=\left\{f_{2}, \ldots, f_{q+1}\right\}$ $\subseteq \mathcal{A}$. Since $F\left(x_{0}\right)=z_{0}$, we have that $f_{2}\left(x_{0}\right)=\ldots=f_{q+1}\left(x_{0}\right)=0$, so $x_{0} \in V(S)$. Notice that

$$
\begin{gathered}
\left(1-f_{1}\left(x_{0}\right)\right) g_{1}\left(x_{0}\right)+\left(0-f_{2}\left(x_{0}\right)\right) g_{2}\left(x_{0}\right)+\ldots+\left(0-f_{q+1}\left(x_{0}\right)\right)=1, \\
1 \cdot g_{1}\left(x_{0}\right)=1, \\
g_{1}\left(x_{0}\right)=1 .
\end{gathered}
$$

But since $K$ is a $q$ th order boundary for $\mathcal{A}$, and since $g_{1} \in \mathcal{A}$, we have that $\exists y_{0} \in K \cap V(S)$ such that

$$
\left|g_{1}\left(y_{0}\right)\right|=\max _{V(S)}\left|g_{1}\right| \geq\left|g_{1}\left(x_{0}\right)\right|=1 \text {. }
$$

Since $y_{0} \in V(S), f_{2}\left(y_{0}\right)=\ldots=f_{q+1}\left(y_{0}\right)=0$; thus since $y_{0} \in K$ we have (from above)

$$
\begin{gathered}
\left\|z_{0}-z_{1}\right\|<(1 / 3) \operatorname{distance}\left(z_{0}, F(K)\right) \leq(1 / 3)\left\|z_{0}-F\left(y_{0}\right)\right\| \\
\|0-(1,0, \ldots, 0)\|<(1 / 3)\left\|0-F\left(y_{0}\right)\right\| \\
1<(1 / 3)\left|f_{1}\left(y_{0}\right)\right|
\end{gathered}
$$

or

$$
\left|f_{1}\left(y_{0}\right)\right|>3 \text {. }
$$


But also

$$
\begin{gathered}
\left(1-f_{1}\left(y_{0}\right)\right) g_{1}\left(y_{0}\right)+f_{2}\left(y_{0}\right) g_{2}\left(y_{0}\right)+\ldots+f_{q+1}\left(y_{0}\right) g_{q+1}\left(y_{0}\right)=1 \\
g_{1}\left(y_{0}\right)=1 /\left(1-f_{1}\left(y_{0}\right)\right) \\
\left|g_{1}\left(y_{0}\right)\right|=1 /\left|f_{1}\left(y_{0}\right)-1\right| \leq 1 /\left(\left|f_{1}\left(y_{0}\right)\right|-1\right)<1 /(3-1)=1 / 2 .
\end{gathered}
$$

Since (2) contradicts (1), we must have that $K$ is a $(q+1)$-tuple boundary for $\mathcal{A}$.

The following corollary is also an immediate consequence of Tonev's results in 7].

Corollary 1. If $\mathcal{F}$ is a uniform algebra with spectrum $M$ and if $K$ is a closed subset of $M$, then $K$ is a qth order boundary for $\mathcal{F}$ if and only if $K$ is a $(q+1)$ tuple boundary for $\mathcal{F}$.

Corollary 2. For $q>0, \partial_{q-1} \mathcal{A}$ is the minimal closed $q$-tuple boundary for $\mathcal{A}$. This is Tonev's result from [7].

However, a $q$ th order boundary for $\mathcal{F}$ need not be a $(q+1)$-tuple boundary for $\mathcal{F}$, even when we make the assumption that $\mathcal{F}$ is a uniform algebra on $M$, if we do not require that the $q$ th order boundary be closed. (For terms and results referenced in the following example, please consult one of the standard texts on uniform algebras such as [3, 4] or [6.)

Example 2. Let $M$ be a compact subset of $\mathbb{C}$, and let $\mathcal{F}$ be the uniform algebra $R(M)$ consisting of the uniform limits of rational functions with poles off $M$. Let $M$ be a "Swiss cheese" or other compact set with empty interior for which, however, $R(M) \neq C(M)$, the algebra of all continuous complex-valued functions on $M$. Let

$$
P=\{x \in M \mid \exists f \in R(M) \text { with } f(x)=1 \text { and }|f|<1 \text { on } M \backslash\{x\}\},
$$

the set of "peak points" for $R(M)$. It is well known that $P$ is a minimal boundary for $R(M)$ in the usual sense of that term (compare [2]).

It follows that

$$
P \text { is a } 0 \text { th order boundary for } R(M) \text {. }
$$

On the other hand, $M$ is the only 1-tuple boundary for $R(M)$. (Consider that $M$ is the one-to-one image of the coordinate function $z$.) Also, $M \backslash P \neq \emptyset$ because $R(M) \neq C(M)$, based on Bishop's peak point criterion for rational approximation. Hence

$$
P \text { is not a 1-tuple boundary for } R(M) \text {. }
$$

In the preceding example, the points of $P$ are the 0-peak points for $R(M)$, while the points of $M$ are the 1-peak points (following Tonev's Definition 3.3.18 in [8].)

Corollary 2 implies that any $q$-tuple boundary for a uniform algebra is dense in $\partial_{q-1} \mathcal{A}$. The following example shows that a $q$-tuple boundary for a uniform algebra can be a proper subset of $\partial_{q-1} \mathcal{A}$.

Example 3. Let $M=\{z:|z| \leq 1\} \times\{t: 0 \leq t \leq 1\}$. Let

$$
\mathcal{A}=\{f: M \rightarrow \mathbb{C} \mid f \text { is continuous, and } f(z, 0) \text { is analytic on }\{|z|<1\}\} \text {. }
$$


Then the minimal boundary for $\mathcal{A}$ in the usual sense is the set of peak points

$$
P=\{|z|=1\} \times\{0\} \cup\{|z| \leq 1\} \times\{t \mid 0<t \leq 1\} .
$$

It is easy to see that in this case $P$ is also a 1-tuple boundary for $\mathcal{A}$.

Finally, we give a result which shows how our notions of boundary apply in the context of linear functions and convex sets rather than uniform algebras. (Compare Tonev's Example 3.2.15 in [8].)

Theorem 2. Let $M$ be a compact, convex subset of a real linear space, and let $\mathcal{L}$ be the space of continuous real linear functions from $M$ to $\mathbb{R}$. Then a set $E \subseteq M$ is a qth order boundary for $\mathcal{L}$ if and only if $E$ is a $(q+1)$-tuple boundary for $\mathcal{L}$.

Proof. A $(q+1)$-tuple boundary for $\mathcal{L}$ is a $q$ th order boundary for $\mathcal{L}$ by Proposition 2 .

Conversely, let $E$ be a $q$ th order boundary for $\mathcal{L}$. Let $F: M \rightarrow \mathbb{R}^{q+1}$, where $F=\left(f_{1}, \ldots, f_{q+1}\right)$ with $f_{1}, \ldots, f_{q+1} \in \mathcal{L}$. Suppose $y \in \partial F(M)$, with say $x \in M$ such that $F(x)=y$. We must show that $\exists \tilde{x} \in E$ with $F(\tilde{x})=y$.

Consider the $2 q+2$ open line segments in $\mathbb{R}^{q+1}$ defined by

$$
\begin{gathered}
L_{0}=y+\{(t, 0, \ldots, 0) \mid t>0\}, \\
L_{1}=y+\{(-t, 0, \ldots, 0) \mid t>0\}, \\
L_{2}=y+\{(0, t, 0, \ldots, 0) \mid t>0\}, \\
\vdots \\
L_{2 q+2}=y+\{(0,0, \ldots, 0,-t) \mid t>0\} .
\end{gathered}
$$

Since $F(M)$ is convex, $\exists j$ such that $L_{j} \subseteq \mathbb{R}^{q+1} \backslash F(M)$, else $y$ would be in the interior of $F(M)$. Say, for example, $L_{0} \subseteq \mathbb{R}^{q+1} \backslash F(M)$. Let $S \subseteq M$ be given by

$$
S=\left\{f_{2}-y_{2}, f_{3}-y_{3}, \ldots, f_{q+1}-y_{q+1}\right\} .
$$

Note that $\# S=q, x \in V(S)$ and $f_{1} \leq y_{1}$ on $V(S)$ by the definition of $L_{0}$.

Let $m=\max _{V(S)}\left(y_{1}-f_{1}\right) \geq 0$, and define $f \in \mathcal{L}$ by

$$
f=f_{1}-y_{1}+m \text {. }
$$

Then $0 \leq f \leq m$ on $V(S)$, because on $V(S)$ we have both

$$
\begin{gathered}
f_{1} \leq y_{1}, \\
f_{1}-y_{1} \leq 0, \\
f \leq m
\end{gathered}
$$

and

$$
\begin{gathered}
y_{1}-f_{1} \leq m, \\
f_{1}-y_{1} \geq-m, \\
f \geq 0 .
\end{gathered}
$$

Since $E$ is a $q$ th order boundary for $\mathcal{L}, \exists \tilde{x} \in E \cap V(S)$ such that

$$
|f(\tilde{x})|=\max _{V(S)}|f|=m .
$$


Since $0 \leq f \leq m$ on $V(S)$, we must have $f(\tilde{x})=m$, so that

$$
f_{1}(\tilde{x})=y_{1}+f(\tilde{x})-m=y_{1} .
$$

Thus

$$
F(\tilde{x})=\left(f_{1}(\tilde{x}), \ldots, f_{q+1}(\tilde{x})\right)=\left(y_{1}, \ldots, y_{q+1}\right)=y,
$$

as desired.

\section{ACKNOWLEDGEMENTS}

The author is grateful to the referee and to John Wermer and Thomas Tonev for their helpful comments, and to the National Science Foundation for its support of his earlier research in this area. This paper is an offshoot of work by the author to prepare a lecture on Uniform Algebras for the Marist College Mathematics Seminar; he wishes to thank the members of the seminar for their encouragement and their interest in this work.

\section{REFERENCES}

[1] R. Basener, A generalized Shilov boundary and analytic structure, Proccedings of the American Mathematical Society 47 (1975), 98-104. MR 50:5476

[2] E. Bishop, A minimal boundary for function algebras, Pacific Journal of Mathematics 9 (1959), 629-642. MR 22:191

[3] A. Browder, Introduction to function algebras, Benjamin, New York, 1969. MR 39:7431

[4] T. Gamelin, Uniform algebras, Prentice-Hall, Englewood Cliffs, New Jersey, 1969. MR 53:14137

[5] N. Sibony, Multi-dimensional analytic structure in the spectrum of a uniform algebra, Springer Lecture Notes in Mathematics, no. 512, Springer Verlag, 1976, pp. 139-175. MR 58:30277]

[6] E. L. Stout, The theory of uniform algebras, Bogden \& Quigley, Inc., Tarrytown-on-Hudson, New York, 1971. MR 54:11066

[7] T. Tonev, New relations between Sibony-Basener boundaries, Springer Lecture Notes in Mathematics, no. 1277, Springer Verlag, 1987, pp. 256-262. MR 89i:46062

[8] _ Big-planes, boundaries and function algebras, Elsevier Science Publishers B.V., Amsterdam, 1992. MR 93h:46065

24 Namoth Road, Wappingers Falls, New York 12590

E-mail address: jbase@idsi.net

E-mail address: basener@alumni.brown.edu 